Category: Teaching \& Learning at times of uncertainty

\title{
Innovative cardiac life support course - hybrid model: international medical university experience during the COVID pandemic
}

\author{
Subramaniam, T., Jee, T.A., Rahman, S.R.A., Goh, A., Asookumaran, S., Alias, A.L., \\ Gangaderan, $S$.
}

International Medical University, Malaysia

\section{Background:}

Curriculum delivery had to continue despite lockdown to achieve clinical learning during the pandemic. Completing the Cardiac Life Support (CLS) course is mandatory to graduate at our university. We restructured the delivery and assessment to ensure that post- lockdown students completed the course. The aim of this study is to determine the outcome of the restructured course on students' performance.

\section{Method:}

A quasi experimental research was conducted on 78 final year medical students; Traditional $(n=28)$ and Hybrid $(n=50)$ ) who completed CLS course at IMU. The traditional CLS course was a full day face to face session but were all converted online using the Microsoft Teams platform. Skills assessment was conducted post- lockdown in the simulation suite adhering to safety protocols and one best answer (OBA) test was conducted online, the maximum mark being 50. Pre-test and post-test scores on OBA and pass /fail in mega-code were analysed using SPSS version 26 . Independent - samples t-test and ANCOVA was utilized for data analysis.

\section{Results:}

Hybrid group did better in the pre-test; $(M=21.7, S D=3.69)$, Traditional; $(M=18.8, S D=3.72) ; t(75)=-$ 3.320, $\mathrm{p}=0.001$ and in the post-test; Hybrid $(\mathrm{M}=26.4, \mathrm{SD}=1.67)$;Traditional $(\mathrm{M}=20.2, \mathrm{SD}=2.53)$; $\mathrm{t}$ $(76)=-13.09, p=<0.001$. ANCOVA test was employed to adjust pre-test scores to 20.64 for all groups and compared with post- course scores; $F(1,74)=137.971 ; p<0.001$. Mega-code passing rate; Hybrid$68 \%(n=34)$; Traditional, 64\% $(n=18)$.

\section{Conclusion:}

Students in the hybrid group did better than those in the traditional group both in knowledge and skills. We believe that this could be the way forward considering the current situation and the unknown future. However, we need to continue this with other cohorts to ensure reproducibility before we can accept this as a substitute for the traditional course.

Keywords: innovation, hybrid, cardiac life support 